\title{
Secukinumab Demonstrated High Effectiveness in Vietnamese Patients with Moderate-To-Severe Plaque Psoriasis in a Real-World Clinical Setting: 16 Week Results from an Observational Study
}

\author{
Hao T. Nguyen (D) - Nhi T. U. Pham • Tu N. A. Tran • Nhuong T. T. Nguyen • \\ Thao T. P. Vu
}

Received: June 15, 2021 / Accepted: July 7, 2021 / Published online: July 18, 2021

(C) The Author(s) 2021

\begin{abstract}
Introduction: Plaque psoriasis (PsO), characterized by demarcated, erythematous, scaly skin, can have a substantial negative impact on patients' quality of life. This observational (noninterventional) retrospective study was conducted to characterize treatment patterns and response among patients with plaque PsO treated with secukinumab under routine medical practice in Vietnam.

Methods: Patient medical records from the specialized clinic of the Ho Chi Minh City Hospital of Dermato-Venereology $(N=236)$ were collected. Patients (male or female) aged $\geq$ 18 years with moderate-to-severe chronic plaque $\mathrm{PsO}$, defined as $>10 \%$ involvement of the body surface area (BSA > 10) or a Psoriasis Area and Severity Index (PASI) score $>10$ and a Dermatology Life Quality Index (DLQI) score $>$ 10 , were included.
\end{abstract}

Results: In total, 230 patients met the inclusion criteria and were included in the intention-to-

H. T. Nguyen $(\bowtie) \cdot$ N. T. U. Pham .

T. N. A. Tran · T. T. P. Vu

Ho Chi Minh City Hospital of Dermato-

Venereology, 2 Nguyen Thong, District 3, Ho Chi

Minh City, Vietnam

e-mail: nthao@ump.edu.vn

N. T. T. Nguyen

Novartis Vietnam Company Limited, Ho Chi Minh

City, Vietnam treat (ITT) population, the majority of whom were men (66.1\%). At baseline, the mean $\pm \mathrm{s}$ tandard deviation (SD) age of the ITT population was $41.46 \pm 14.29$ years, mean disease duration was $7.91 \pm 7.91$ years, and $27 \%$ $(n=62)$ were obese. More than $90 \%$ of the patients were biologic naïve prior to initiation of secukinumab therapy. At week 4, 54.6\% patients $(n=124)$ achieved $\geq 75 \%$ reduction in PASI scores from baseline (PASI 75). By week 16, $81.1,68.9$, and $36.5 \%$ of the overall population $(n=180,153$, and 81$)$ achieved PASI scores of 75,90 , and 100 , respectively; $66.1 \%$ of the overall population $(n=154)$ reported DLQI scores of $0 / 1$ by week 16 . The effectiveness of secukinumab was validated in subgroups of patients with or without obesity, concomitant conditions (hepatitis B virus, hepatitis $C$ virus, diabetes, high blood pressure, gout, and/or obesity [body mass index $\geq 30 \mathrm{~kg} / \mathrm{m}^{2}$ ]), and concomitant psoriatic arthritis (PsA).

Conclusion: The study validated the real-world effectiveness of secukinumab in Vietnamese patients irrespective of obesity, concomitant conditions, and concomitant PsA status.

Keywords: Effectiveness; Psoriasis; Real-world; Secukinumab; Vietnam 


\section{Key Summary Points}

Secukinumab has proven to be highly efficacious in the long-term treatment of the key manifestations of psoriatic disease with a favorable safety profile.

Evidence on the real-world effectiveness and safety of secukinumab in Vietnamese population is lacking, emphasizing the need to assess the use of secukinumab for the treatment of psoriasis in daily practice and validate the impact on psoriasis disease activity in real-life in Vietnamese patients.

By week 16 of treatment, 81.1, 68.9, and $36.5 \%$ of the overall patient population achieved PASI 75, 90, and 100, respectively.

Clinical effectiveness of secukinumab was complemented by the improvement in quality of life, with $66.1 \%$ patients achieving a Dermatology Life Quality Index score of $0 / 1$ by week 16 .

The results validate the real-world effectiveness of secukinumab in Vietnamese patients irrespective of obesity, concomitant conditions, and concomitant psoriatic arthritis status.

\section{INTRODUCTION}

Immune-mediated inflammatory disorders are a diverse group of conditions resulting from an imbalance in inflammatory cytokine activities [1]. Psoriatic disease is a common immune-mediated inflammatory disorder that presents different clinical manifestations, including plaque psoriasis (PsO) and psoriatic arthritis (PsA). Plaque PsO, which affects approximately $85-90 \%$ of patients with psoriatic disease [2], is characterized by demarcated, erythematous, scaly skin $[3,4]$ and can have a substantial negative impact on patients' quality of life (QoL) [5].

A recent systematic review on the prevalence of psoriasis worldwide published in 2020 estimated that there were 288,580 cases of adult psoriasis in the general Vietnamese population; this is equivalent to a crude lifetime prevalence (CLT, physician or dermatologist diagnosed) estimate of 0.30 and is lower than the estimated 0.47 for the global population [6]. In terms of onlyadult patients, the CLT was estimated to be 0.38 and 0.59 for Vietnam and the world population, respectively [6].

Secukinumab, a fully human monoclonal antibody that selectively neutralizes interleukin-17A, has proven highly efficacious in the long-term treatment of the key manifestations of psoriatic disease with a favorable safety profile [7-10]. The efficacy and safety of secukinumab in moderate-to-severe plaque $\mathrm{PsO}$ has been proven in two large phase 3 studies, ERASURE and FIXTURE 1, and several other clinical trials [7-10]. In these trials, patients treated with secukinumab showed substantial improvements in core parameters of disease activity, such as the Psoriasis Area and Severity Index (PASI) score and Dermatology Life Quality Index (DLQI) score, under randomized controlled trial (RCT) conditions compared with ustekinumab [9].

Real-world clinical practice for the treatment of secukinumab is significantly different from that applied in RCTs with strictly regulated inclusion/exclusion criteria [11]. Garcia-Doval and colleagues reported that up to $30 \%$ of psoriasis patients in a real-world setting would be ineligible for inclusion in RCTs [12]. This, in turn, might affect the treatment outcomes of secukinumab in the real-world clinical setting. The real-world effectiveness and safety of secukinumab were demonstrated in the PROSPECT study as well as in Japanese and Taiwanese populations [11, 13-15]. Epidemiology data suggest that $\mathrm{PsO}$ is more prevalent in Vietnam $(2.2 \%)$ than in Taiwan $(0.2 \%)$ and Japan (0.3\%) [16-18]. Unlike high-income countries such as Taiwan and Japan, Vietnam is a lower-middle-income country where although secukinumab was approved in 2016, it is only since 2019 that is partially reimbursed. 
Consequently, due to the high cost of the drug and the required co-payments, patient access to biologic therapy is limited. Thus, the real-world data from Japan or Taiwan may not be generalizable to the Vietnamese population.

To complement the current body of evidence on the real-world use of secukinumab, we have carried out a study aimed to assess the use of secukinumab in daily practice of $\mathrm{PsO}$ treatment and validate the impact on $\mathrm{PsO}$ disease activity in real-life in Vietnamese patients.

\section{METHODS}

\section{Study Design}

This was an observational (non-interventional) retrospective study to characterize treatment patterns and response under routine medical practice in Vietnam among patients with plaque $\mathrm{PsO}$ conducted at Ho Chi Minh City Hospital of Dermato-Venereology (HHDV). Patients were followed up for at least 16 weeks from enrollment.

The primary objective of this study was to assess the proportion of patients achieving PASI 75 (75\% reduction in disease activity) at week 16 from baseline with real-life treatment with secukinumab at the HHDV. Secondary objectives determining the proportion of patients achieving PASI 90/100 (90/100\% reduction in disease activity from baseline) and DLQI score $0 / 1$ (= no effect of disease on patient's life) at week 16 and the proportion of patients achieving PASI 75 at week 4, and characterizing the profile of patients with plaque $\mathrm{PsO}$ treated with secukinumab at the HHDV.

This study has been reviewed and approved by the Institutional Review Board/Independent Ethics Committee of the HHDV (Approval Number: 141/QĐ-BVDL, date 17 March 2020) and competent authority (CA) according to national and international regulations. Informed consent was waived due to the retrospective nature of the study.

\section{Study Population}

Patients (male or female) aged $\geq 18$ years with moderate-to-severe chronic plaque $\mathrm{PsO}$ were included in this study. Moderate-to-severe chronic plaque $\mathrm{PsO}$ was defined by $>10 \%$ involvement of the body surface area (BSA $>10$ ) or a PASI score $>10$ on a scale of $0-72$ and a DLQI score $>10$ on a scale of $0-30$. Included patients were required to be managed and treated with at least one dose of secukinumab at the HHDV at least 16 weeks before the enrollment date. Patients who did not have a medical record of $\mathrm{PsO}$ or were participating in other clinical trials that may interfere with the progress of this study were excluded.

\section{Data Collection}

A specialized clinic of the HHDV has been running a PsO treatment program since November 2017. For the purpose of this study, data were extracted from the patient medical records of this program since its inception. The data collected through the patient medical chart may include patients on secukinumab maintenance treatment who had started secukinumab treatment prior to November 2017.

Patient demographics, disease characteristics (e.g., duration of PsO, concomitant PsA, comorbidities, and $\mathrm{PsO}$ in specific areas), and disease severity (e.g., PASI and DLQI scores) were recorded.

\section{Statistical Analysis}

Statistical analysis was conducted using R statistical software (SAS Institute, Cary, NC, USA), and a $P$ value of $<0.05$ was considered statistically significant. All missing effectiveness data at different time points were subject to as-treated (AT) and non-responder imputation (NRI) analyses. The clinical response rates achieved by the different subgroups of patients were compared using a Chi-square test. 


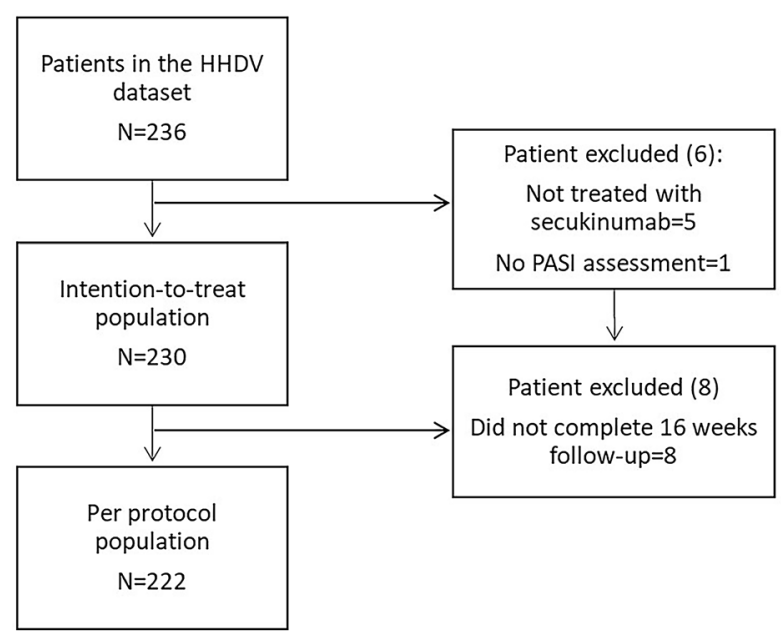

Fig. 1 Flow chart of patient enrollment. $H H D V$ Ho Chi Minh City Hospital of Dermato-Venereology, $N$ total number of patients, PASI Psoriasis Area and Severity Index

Analysis was performed descriptively and continuous variables were reported as mean, SD, median and 25th and 75th quartiles, and range. Categorical variables were reported as number and proportion of patients with observed (non-missing) data.

\section{RESULTS}

\section{Demographics and Baseline Characteristics}

In total, the HHDV dataset contained 236 records of patients with plaque PsO (Fig. 1). Of these, 230 patients were treated with secukinumab and the PASI score at baseline was available; these 230 patients were included in the intention-to-treat (ITT) population. Among the ITT population, 222 patients completed the 16-week follow-up and were included in the per protocol (PP) population.

In the ITT population, the majority of patients were men $(66.1 \%)$, and at baseline the mean age \pm standard deviation (SD) was $41.46 \pm 14.29$ years, mean body mass index (BMI) was $23.47 \pm 4.70 \mathrm{~kg} / \mathrm{m}^{2}$, and $27 \%$ of patients $(n=62)$ were obese (Table 1$)$. The mean disease duration of patients was $7.91 \pm$ 7.91 years. A total of $30.9 \%$ of patients $(n=71)$ in the ITT population had comorbidities at baseline, of which the most commonly reported were obesity $(27.0 \%)$, high blood pressure (5.7\%), and hepatitis B virus infection (3.5\%). Up to $51.3 \%$ of patients $(n=118)$ reported overall nail manifestation (including onycholysis, pitting, subungual hyperkeratosis, and other nail changes), while $7.0 \%(n=16)$ reported concomitant PsA. The majority of the patients were biologic naïve $(96.5 \%)$. Patient demographics and disease characteristics at baseline are presented in Table 1.

\section{Treatment Effectiveness}

In this study, secukinumab treatment was highly effective in patients with moderate-tosevere $\mathrm{PsO}$ as early as 4 weeks following initiation of treatment, and this was sustained up to 16 weeks, as demonstrated by the PASI and DLQI responses. At week 4, 54.6\% of patients $(n=124)$ achieved PASI 75 response compared to none at baseline. By week 16, 81.1, 68.9, and $36.5 \%$ of the overall ITT population $(n=180$, 153 , and 81) achieved PASI 75, 90, and 100, respectively. Improvement in disease activity was complemented by overall improvement seen in QoL assessment, with $66.1 \%$ of the overall population $(n=154)$ reporting DLQI $0 / 1$ score by week 16 (Fig. 2).

The aim was to identify the predictors of response by stratifying patients based on obesity 
Table 1 Demographics and disease characteristics of the intention-to-treat study population at baseline

\begin{tabular}{|c|c|}
\hline Characteristics & Values $(N=230)^{\mathrm{a}}$ \\
\hline \multicolumn{2}{|l|}{ Gender } \\
\hline Male & $152(66.1)$ \\
\hline Female & $78(33.9)$ \\
\hline Age (year) & $41.46 \pm 14.29$ \\
\hline Weight $(\mathrm{kg})$ & $63.5 \pm 14.3$ \\
\hline Height $(\mathrm{cm})$ & $163.8 \pm 8.1$ \\
\hline BMI $\left(\mathrm{kg} / \mathrm{m}^{2}\right)$ & $23.47 \pm 4.70$ \\
\hline Duration of disease (year) & $7.91 \pm 7.91$ \\
\hline Comorbidities (yes) & $71(30.9)$ \\
\hline HBV & $8(3.5)$ \\
\hline $\mathrm{HCV}$ & $2(0.9)$ \\
\hline Diabetes & $6(2.6)$ \\
\hline High blood pressure & $13(5.7)$ \\
\hline Gout & $2(0.9)$ \\
\hline Obesity $\left(\mathrm{BMI} \geq 30 \mathrm{~kg} / \mathrm{m}^{2}\right)$ & $62(27.0)$ \\
\hline \multicolumn{2}{|l|}{$\mathrm{PsO}$ at special areas (yes) } \\
\hline Face & $14(6.1)$ \\
\hline Genitalia & 0 \\
\hline Hand & 0 \\
\hline Foot & $1(0.4)$ \\
\hline \multicolumn{2}{|c|}{ Presence of joints and nails manifestations (yes) } \\
\hline Joint swelling & $12(5.2)$ \\
\hline Joint pain/tenderness & $24(10.4)$ \\
\hline Joint deformity & $5(2.2)$ \\
\hline Onycholysis & $35(15.2)$ \\
\hline Nail pitting & $74(32.2)$ \\
\hline Hyperkeratosis & $8(3.5)$ \\
\hline Nail changes & $27(11.7)$ \\
\hline Overall nail $\mathrm{PsO}_{s}$ & $118(51.3)$ \\
\hline PsA & $16(7.0)$ \\
\hline
\end{tabular}

Table 1 continued

\begin{tabular}{ll}
\hline Characteristics & Values $(\boldsymbol{N}=\mathbf{2 3 0})^{\mathbf{a}}$ \\
\hline Previous biologic & \\
Yes & $8(3.5)$ \\
No & $222(96.5)$ \\
\hline
\end{tabular}

$B M I$ Body mass index, $H B V$ Hepatitis $\mathrm{B}$ virus, $H C V$ Hepatitis $\mathrm{C}$ virus, ITT intention-to-treat, $P_{S} A$ psoriatic arthritis, $P_{s} O$ psoriasis

${ }^{a}$ Values in table are presented as the mean \pm standard deviation (SD) or as the frequency ( $n$, number of patients) with the percentage in parentheses, as appropriate

status, presence of comorbidities, and presence of PsA. Similar improvement in disease activity and QoL was observed when stratification was applied.

A total of $80.0,63.3$, and $23.3 \%$ of patients ( $n=48,38$, and 14) with obesity at baseline achieved PASI 75, 90, and 100, respectively, at week 16 (vs. none at baseline). Similarly, for non-obese patients, 81.5, 71.0, and $41.4 \%$ $(n=132,115$, and 67$)$ achieved PASI 75, 90, and 100 , respectively, at week 16 . In addition, $63 \%$ of patients $(n=39)$ with obesity at baseline and $67.7 \%$ of non-obese patients $(n=115)$ achieved DLQI 0/1 by week 16 (Fig. 3a, b).

When stratified by comorbidity, at week 16 , $82.4,52.9$, and $35.3 \%$ of patients with a comorbidity ( $n=14,9$, and 6 ) and 81.0, 70.2, and $36.6 \%$ of those without comorbidity $(n=166,144$, and 75$)$ achieved PASI 75, 90, and 100 , respectively. Similarly, improvement in DLQI was seen in both subgroups of patients (63.2 and $67.3 \% ; n=13$ and 141 , respectively) (Fig. 3c, d).

When stratified by concomitant PsA, at week $16,75.0,62.5$, and $25.0 \%$ of patient with concomitant PsA ( $n=12,10$, and 4$)$ and 81.6, 69.4, and $37.4 \%$ of those without concomitant PsA ( $n=168,143$, and 77) achieved PASI 75, 90, and 100 , respectively. Similarly, improvement in DLQI was seen in both subgroups of patients (43.8 and $67.8 \% ; n=8$ and 146 , respectively) (Fig. 3e, f). 


\section{Overall Population}

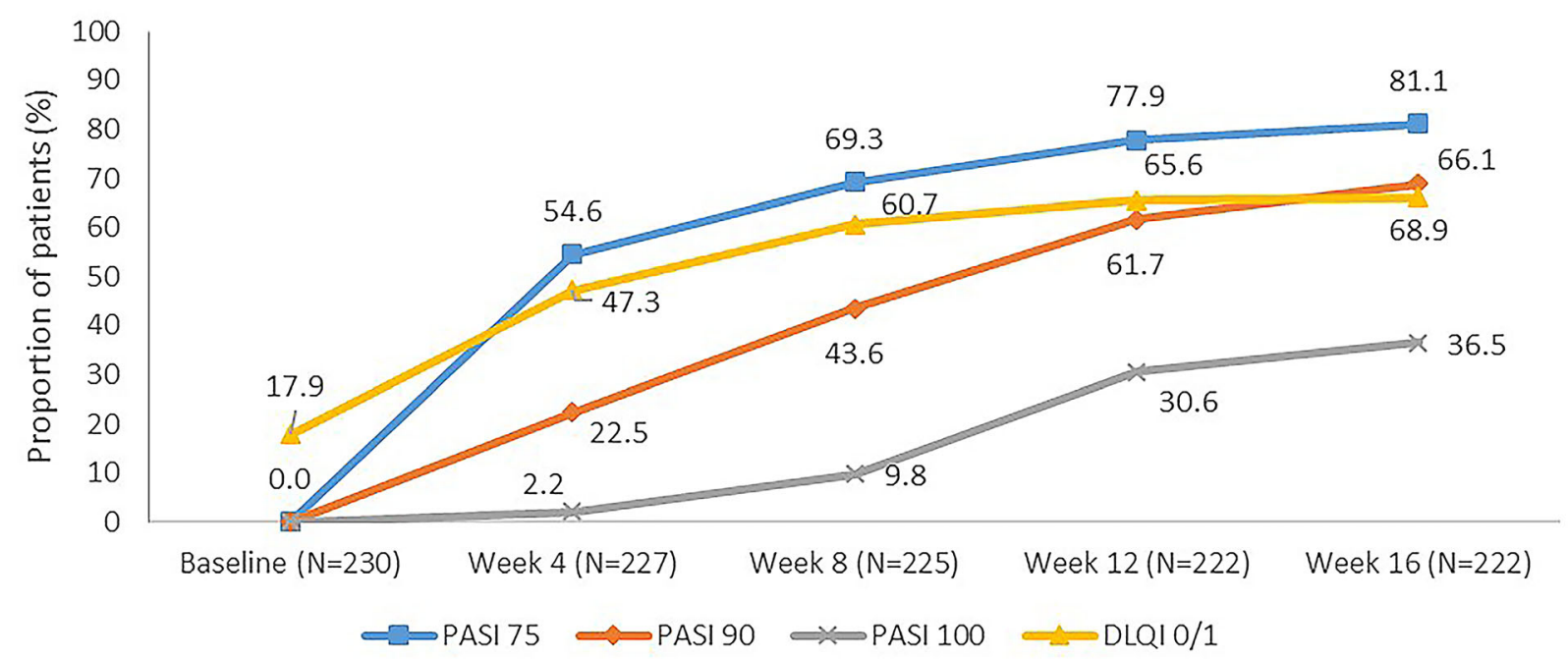

Fig. 2 Disease activity and quality of life assessment over time in the intention-to-treat study population. DLQI $0 / 1$ Dermatology Life Quality Index score indicating no effect of disease on patient's life, ITT intention-to-treat,

\section{DISCUSSION}

To our knowledge, this is the first study to report the real-world effectiveness of secukinumab in Vietnam. The results will contribute to the body of evidence on the use of secukinumab in daily practice of PsO treatment and validate the impact on $\mathrm{PsO}$ disease activity in real-life in Vietnamese patients.

Our findings demonstrate the effectiveness of secukinumab in Vietnamese patients with moderate-to-severe $\mathrm{PsO}$ and are in line with those observed in the global population [7-9]. The early clinical effect of secukinumab was shown by the high proportion of Vietnamese patients achieving PASI 75, 90, and 100 as early as week 4; improvement continued over time up to week 16. PASI 75 has been considered to indicate treatment success for the treatment of PsO [19]. In the current study, $81.1 \%$, of Vietnamese patients achieved the primary endpoint of PASI 75, consistent with the findings reported in pivotal trials (67.0-81.6\%) [7-9] as well as in regional real-world studies ( $>$ 80.3-83\%) $[15,20]$. PASI 90 and 100 response $(68.9 \%$ and
$N$ number of patients, PASI 75, 90, 100 PASI scores indicating 75,90 , and $100 \%$ improvement in disease activity from baseline, respectively, QoL quality of life

$36.5 \%)$ were also consistent with earlier trials (PASI $90=62-65.7 \%$; PASI $100=44.5$ ) $[15,20]$.

The clinical effectiveness of secukinumab demonstrated in our ITT population was complemented by the improvement in QoL, with $66.1 \%$ patients achieving DLQI $0 / 1$ by week 16 , similar to values observed in other real-world studies (61.2-71.9\%) [9, 14, 15]. QoL was improved in line with clinical efficacy, reaching a plateau at around week 12 .

A previous study demonstrated that the pharmacokinetics of secukinumab was affected by body weight [21]. Two real-world studies from Spain reported that a significantly higher proportion of patients with $\mathrm{BMI}<30 \mathrm{~kg} / \mathrm{m}^{2}$ achieved PASI 75 as compared with patients with $\mathrm{BMI} \geq 30 \mathrm{~kg} / \mathrm{m}^{2}[20,22]$. The same has been reported for subgroup analyses of the ERASURE and FIXTURE studies [23]. This is in contrast to the current study, in which similar effectiveness was observed in both groups. It is worth noting that in both the Notario et al. [22] and Ortiz-Salvador et al. [20] studies, a higher percentage of patients were obese compared to current study (44.9 vs. $27.0 \%$ ). The lower proportion of obese patients in the current study 

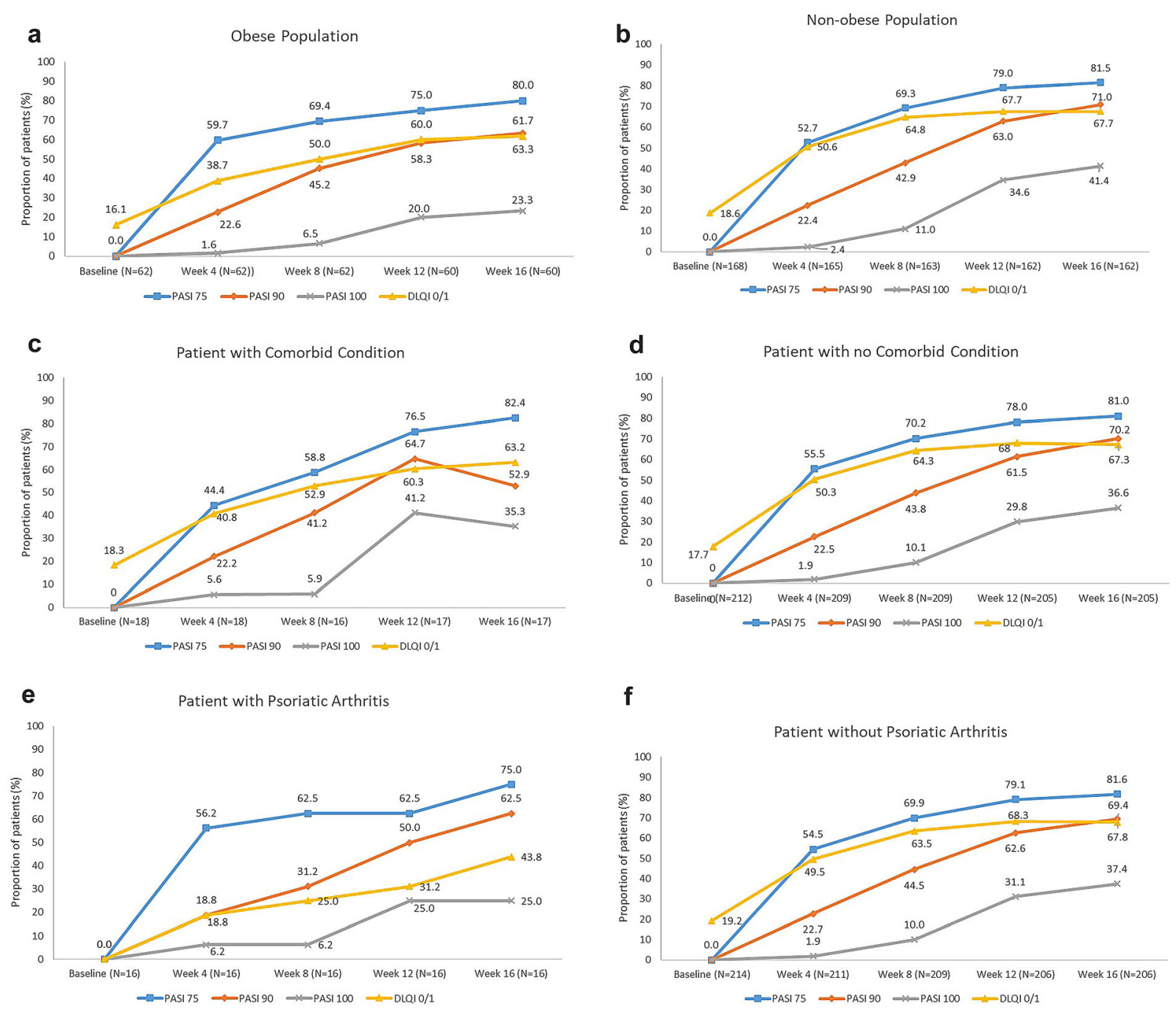

Fig. 3 Clinical activity and QoL assessment of patients over time stratified by obesity status (a, b), presence of comorbid conditions $(\mathbf{c}, \mathbf{d})$, and concomitant PsA $(\mathbf{e}, \mathbf{f}) . N$ Number of patients, $P s A$ psoriatic arthritis

may be reflective of the unique patient profiles for PsO in Vietnam and contribute to the results observed in this study. The current study also validated the effectiveness of secukinumab in subgroups of patients with or without comorbidities, as well as concomitant PsA, similar to an earlier study [22].

Failure of prior biologic therapies was demonstrated to be a response modifier in Japanese and Spanish patients [15, 20]. However, this was not a reported parameter in the current study. Patients recruited in this Vietnamese real-world study had shorter mean disease duration (7.9 years) compared with a realworld study reported previously $[15,22]$ but had overall more severe disease, as indicated by the baseline PASI score (21.9) versus the two realworld Spanish trials (12.5-13.5) [20, 22]. Higher absolute PASI at baseline may be an indicator that most patients in the current study had no prior systemic therapies. The possibility of prior biologic therapies failure as a response modifier in Vietnamese patients warrants further study.

This was an observational study conducted in a PsO-specific clinic and, consequently, may be subject to confounding and a variety of biases. The current study had a low patient number and short treatment follow-up period. However, there were adequate data to stratify patients by comorbidity and concomitant PsA, and the 
authors are hopeful that the result can add valuable information in terms of the effectiveness of secukinumab in real-world practice.

\section{CONCLUSION}

In conclusion, the study validated the effectiveness of secukinumab in Vietnamese patients irrespective of obesity, comorbidities, and concomitant PsA status.

\section{ACKNOWLEDGEMENTS}

Funding. The study is a research collaboration between Novartis Vietnam Co., Ltd and HHDV. This study and the journal's Rapid Service Fee were funded by Novartis Vietnam Co., Ltd and HHDV is the regulatory sponsor.

Medical Writing, Editorial and Other Assistance. The authors would like to thank Yen Thi Bui (Novartis Vietnam Co., Ltd.), Poh Sien Ooi (Novartis Corporation Sdn Bhd), and Avishek Anant (Novartis Healthcare Pvt Ltd) for providing medical writing assistance in accordance with the Good Publication Practice (GPP3) guidelines (http://www.ismpp.org/gpp3).

Authorship. All named authors meet the International Committee of Medical Journal Editors (ICMJE) criteria for authorship for this article, take responsibility for the integrity of the work as a whole, and have given their approval for this version to be published. Authors also take complete responsibility for the integrity of the data and accuracy of the data analysis. The authors thank Dung Van Do for being medical consultant and providing data analysis of the study. All authors take responsibility for the accuracy of the results. All authors reviewed and provided feedback on subsequent versions and agreed on the final version and to submit the manuscript for publication.

Author Contributions. Study conception and design: Hao T Nguyen; data collection: Nhi
TU Pham, Tu NA Tran, Thao TP Vu; data analysis and interpretation: Hao T Nguyen, Nhi TU Pham, Tu NA Tran, Nhuong TT Nguyen, Thao $\mathrm{TP} \mathrm{Vu}$; manuscript drafting and revision: Hao T Nguyen, Nhi TU Pham, Tu NA Tran, Nhuong TT Nguyen, Thao TP Vu.

Disclosures. Hao T Nguyen has served as an advisory board member and speaker for Novartis, Janssen, and Menarini. Nhi TU Pham, Tu NA Tran, and Thao TP Vu have served as a speaker for Novartis, Janssen, and Menarini. Nhuong T Nguyen is an employee of Novartis Vietnam Co., Ltd.

Compliance with Ethics Guidelines. This study has been reviewed and approved by the Institutional Review Board/Independent Ethics Committee of the HHDV (Approval Number: 141/QĐ-BVDL, date 17 March 2020) and competent authority (CA) according to national and international regulations. Informed consent was waived for the retrospective nature of the study.

Data Availability. The datasets generated during and/or analyzed during the current study are available from the corresponding author on reasonable request.

Open Access. This article is licensed under a Creative Commons Attribution-NonCommercial 4.0 International License, which permits any non-commercial use, sharing, adaptation, distribution and reproduction in any medium or format, as long as you give appropriate credit to the original author(s) and the source, provide a link to the Creative Commons licence, and indicate if changes were made. The images or other third party material in this article are included in the article's Creative Commons licence, unless indicated otherwise in a credit line to the material. If material is not included in the article's Creative Commons licence and your intended use is not permitted by statutory regulation or exceeds the permitted use, you will need to obtain permission directly from the copyright holder. To view a copy of this licence, visit http://creativecommons.org/licenses/by$\mathrm{nc} / 4.0 /$. 


\section{REFERENCES}

1. Williams JP, Meyers JA. Immune-mediated inflammatory disorders (I.M.I.D.s): the economic and clinical costs. Am J Manag Care. 2002;8:S664-81.

2. Boehncke W, Schön MP. Psoriasis. Lancet. 2015;386:983-94.

3. Campa M, Mansouri B, Warren R, Menter A. A review of biologic therapies targeting IL-23 and IL17 for use in moderate-to-severe plaque psoriasis. Dermatol Ther (Heidelb). 2016;6(1):1-12.

4. Nestle FO, Conrad C, Tun-kyi A, et al. Plasmacytoid predendritic cells initiate psoriasis through interferon-production. J Exp Med. 2005;202(1):135-43.

5. Langley RGB, Krueger GG, Griffiths CEM. Psoriasis: epidemiology, clinical features, and quality of life. Ann Rheum Dis. 2005;64(Suppl II):18-23.

6. Parisi R, Iskandar IYK, Kontopantelis E, et al. National, regional, and worldwide epidemiology of psoriasis: systematic analysis and modelling study. BMJ. 2020;369:m1590.

7. Langley R, Elewski B, Lebwohl M, et al. Secukinumab in plaque psoriasis-results of two phase 3 trials. N Engl J Med. 2014;371:326-38.

8. Gottlieb AB, Langley RG, Philipp S, et al. Secukinumab improves physical function in subjects with plaque psoriasis and psoriatic arthritis: results from two randomized, phase 3 trials. J Drugs Dermatol. 2015;14(8):821-33.

9. Thaci D, Blauvelt A, Reich K, et al. Secukinumab is superior to ustekinumab in clearing skin of subjects with moderate to severe plaque psoriasis: CLEAR, a randomized controlled trial. J Am Acad Dermatol. 2015;73(3):400-9.

10. Bagel J, Nia J, Hashim PW, et al. Secukinumab is superior to ustekinumab in clearing skin in patients with moderate to severe plaque psoriasis (16-Week CLARITY results). Dermatol Ther. 2018;8(4):571-9.

11. Ger T, Huang Y, Hui RC, Tsai T-F, Chiu H-Y. Effectiveness and safety of secukinumab for psoriasis in real-world practice: Analysis of subgroups stratified by prior biologic failure or reimbursement. Ther Adv Chronic Dis. 2019;10:1-9.

12. Garcia-Doval I, Carretero G, Vanaclocha F, Ferrandiz C, Daude E. Risk of serious adverse events associated with biologic and nonbiologic psoriasis systemic therapy. Arch Dermatol. 2012;148(4): 463-70.
13. Deodhar A, Mease PJ, McInnes IB, et al. Long-term safety of secukinumab in patients with moderateto-severe plaque psoriasis, psoriatic arthritis, and ankylosing spondylitis: Integrated pooled clinical trial and post-marketing surveillance data. Arthritis Res Ther. 2019;21(1):111.

14. Thaci D, Korber A, von Kiedrowski R, et al. Secukinumab is effective in treatment of moderate-tosevere plaque psoriasis: real-life effectiveness and safety from the PROSPECT study. JEADV. 2020;34: 310-8.

15. Fujita H, Ohtsuki M, Morita A, et al. Safety and effectiveness of secukinumab in psoriasis vulgaris and psoriatic arthritis: real-world evidence in Japan. J Dermatol. 2021;48(2):175-83.

16. Ha VS, Nguyen CTT, Nguyen TTT. Cost-effectiveness of biological drugs for the treatment of moderate-to-severe plaque psoriasis: a systematic review. Rev Medicale. 2019;1:112-22.

17. Chang Y, Chen T, Liu P, Chen Y, Chen Y, Huang Y, et al. Epidemiological study of psoriasis in the National Health Insurance database in Taiwan. Acta Derm Venereol. 2009;89:262-326.

18. Kubota K, Kamijima Y, Sato T, et al. Epidemiology of psoriasis and palmoplantar pustulosis: a nationwide study using the Japanese national claims database. BMJ Open. 2015;5:e006450.

19. Mrowietz U, Kragballe K, Reich K, et al. Definition of treatment goals for moderate to severe psoriasis: A European consensus. Arch Dermatol Res. 2011;303(1):1-10.

20. Ortiz-Salvador J-M, Saneleuterio-Temporal M, Magdaleno-Tapial J, et al. A prospective multicenter study assessing effectiveness and safety of secukinumab in a real-life setting in 158 patients. J Am Acad Dermatol. 2019;81(2):427-32.

21. Bruin G, Loesche C, Nyirady J, Sander O. Population pharmacokinetic modeling of secukinumab in patients with moderate to severe psoriasis. J Clin Pharmacol. 2017;57(7):876-85.

22. Notario J, Deza G, Vilarrasa E, et al. Treatment of patients with plaque psoriasis with secukinumab in a real-life setting: A 52-week, multicenter, retrospective study in Spain. J Dermatolog Treat. 2019;30(5):424-9.

23. Szepietowski J, Rich P, Loeffler J, et al. Secukinumab $300 \mathrm{mg}$ shows superior efficacy across subject body weight groups: pooled analysis of phase 3 ERASURE and FIXTURE trials. J Am Acad Dermatol. 2015;72(5):AB248. 